\title{
MESURES CONIQUES ET INTÉGRALE DE DANIELL
}

\author{
RICHARD BECKER
}

(Received 25 February 1980; revised 24 September 1980)

Communicated by R. O. Vyborny

\begin{abstract}
Let $X$ be a weakly complete proper cone contained in a weak space $E$ and $h(E)$ the Riesz space generated by the continuous linear forms on $E$. A positive conical measure $\mu$ on $X$ is a positive linear form on $\left.h(E)\right|_{X}$. G. Choquet has proved $\mu$ is a Daniell integral on $E$ when $E$ is weakly complete, but $\mu$ is not generally a Daniell integral on $X$. However we give an integration theory for functions on $X$ and compare this theory with the classical Daniell theory. The case where $\mu$ is maximal in the sense of $\mathrm{G}$. Choquet is remarkable.
\end{abstract}

1980 Mathematics subject classification (Amer. Math. Soc.): 28 A 25.

\section{Préliminaires}

1. Notations et rappels. L'expression " $E$ espace faible" signifie que $E$ est un espace vectoriel muni d'une topologie faible séparée; $E^{\prime}$ désigne le dual de $E$ pour cette topologie; quelquefois, il pourra arriver qu'un même espace vectoriel $E$ soit mis en dualité avec plus d'un s.e.v. de $E^{*}$ (dual algébriqud de $E$ ): cela sera alors explicitement précisé.

On dit qu'un cóne convexe, contenu dans un espace faible $E$, est dans la classe $\mathcal{C}$ lorsque ce cône est une partie complète de $E ; \mathcal{\delta}$ désigne la classe des éléments saillants de $\mathcal{C}$.

Rappelons que, si $V$ est un espace vectoriel ordonné, $V^{+}$désigne le cône des éléments $\geqslant 0$ de $V$, et $V_{b}^{*}$ le s.e.v. de $V^{*}$ engendré par $\left(V^{*}\right)^{+}$.

Soit $E$ espace faible; on note $h(E)$ l'espace vectoriel réticulé (e.v.r.) de fonctions définies sur $E$ engendré par $E^{\prime}$, et $S(E)$, la famille des éléments convexes de $h(E)$.

(C) Copyright Australian Mathematical Society 1981 
On pose $M(E)=h(E)_{b}^{*}$; les éléments de $M(E)$ sone appelés mesures coniques sur $E$. On dit que $\mu \in M^{+}(E)$ est portée par un cône $X \subset E$ lorsque $(f \in h(E)$ et $\left.\left.f\right|_{X}>0\right)$ entraine $(\mu(f)>0) ; \mu(f)$ ne dépend alors que de $\left.f\right|_{X}$. On dit que $\mu \in M(E)$ est portée par $X$ si, et seulement si, $\mu^{+}$et $\mu^{-}$sont portées par $X$.

Soient $E$ espace faible, et $X \in \mathcal{C}$ avec $X \subset E ; \forall \mu \in M^{+}(E)$, lorsque $\mu$ est portée par $X$, il existe un élément $r(\mu) \in X$ tel que, $\forall l E^{\prime}, \mu(l)=l(r(\mu))([4]$, 30.7 proposition); on dit que $r(\mu)$ est la résultante de $\mu$. On pose

$$
K_{\mu}=\left(r(\lambda) ; \lambda \in M^{+}(E) \text { avec } \lambda<\mu\right) ;
$$

c'est une partie convexe et compacte de $X$ ([4], 38.2 proposition).

Il existe un plus petit élément de $\mathcal{C}$ qui porte $\mu$, à savoir $\left(\overline{\cup n K_{\mu}}\right)([4], 38.5$ corollaire).

Soient $E$ espace faible, $X \in \mathcal{C}$ avec $X \subset E$, et $\lambda, \mu \in M^{+}(E), \lambda$ et $\mu$ étant portées par $X$. On dit que $\lambda$ est plus concentrée que $\mu$, et on note $\lambda \prec \mu$ lorsque, $\forall f \in S(E), \lambda(f)<\mu(f)$ (ordre de Choquet) ([4] 30.11). On appelle mesure maximale sur $X$ toute mesure maximale pour cet ordre ([4] 30.13). Il est évident que s'il existe une mesure maximale sur $X$, alors $X \in \mathcal{E}$.

2. Introduction. G. Choquet ([4] 38.13) a démontré qu'un mesure conique $\mu>0$ sur un espace $E$ faiblement complet (i.e. $E \in \mathcal{C}$ ) était une intégrale de Daniell (i.e. $\forall\left(f_{n}\right) \in h(E)$, avec $f_{n} \downarrow 0$ sur $E$, on a $\mu\left(f_{n}\right) \rightarrow 0$ ).

Mais $\mu$ n'est pas nécessairement une intégrale de Daniell pour la convergence sur un cône $X \in \mathcal{C}$ qui porte $\mu$ (cf. $n^{\circ} 8$ ). Le problème se pose alors de tenter d'édifier une théorie de l'intégration pour une mesure conique $>0$ portée par un cône donné de la classe $\mathcal{C}$, et de comparer cette théorie avec la théorie classique de Daniell.

3. Plan. Après ces quelques préliminaires (partie I), et étant donné un espace faible $E$, et $X \in \mathcal{C}$ avec $X \subset E$, nous montrerons, dans la partie II, que, pour ainsi dire, la notion de mesure conique portée par $X$ ne change pas lorsque l'on renforce la topologie de $E$ en rajoutant à $E^{\prime}$ des éléments de $E^{*}$ dont la restriction à $X$ est continue.

Soient $E$ espace faible, $X \in \mathcal{C}$ avec $X \subset E$, et $\mu \in M^{+}(E)$ portée par $X$.

Nous définirons alors (partie III) l'analogue de l'intégrale supérieure de la théorie classique, pour des fonctions sous-linéaires et s.c.i., définies sur $X\left(n^{\circ} 12\right)$; nous prouverons une propriété de continuité pour l'intégrale supérieure $\left(n^{\circ} 17\right)$, grâce à un "lemme de Fatou conique" $\left(n^{\circ} 9\right)$.

Nous introduirons un espace vectoriel réticulé $\Lambda^{1}(\mu)\left(n^{\circ} 18\right)$ de fonctions sur $K_{\mu}$ contenant $h(E)$, sur lequel un prolongement de $\mu$ est défini grâce à l'intégrale supérieure, et nous comparerons $\Lambda^{1}(\mu)$ avec l'espace $L^{1}(\mu)$ déduit de $\mu$ et de 
$h(E)$ à l'aide de la théorie classique de Daniell ( $n^{\circ} 21$ et 23). Le cas où $X \in \mathcal{S}$ et où $\mu$ est maximale est particulièrement intéressant $\left(n^{\circ} 19\right)$.

Enfin dans la partie IV, nous prolongerons $\mu$ aux fonctions sous-linéaires définies sur $X\left(n^{\circ} 25\right)$; on prouvera une propriété de continuité séquentielle de ce prolongement $\left(n^{\circ} 29\right)$,

\section{Une remarque concernant la définition des mesures coniques}

Soit $X$ un cône convexe contenu dans un espace vectoriel; on pose $E=X-$ $X$, et on suppose que $X \in \mathcal{C}$ lorsque $E$ est mis en dualité avec un certain espace vectoriel $F \subset E^{*}$. Il est bien connu ([2] chap. II, \$3, p. 7) que $X \in \mathcal{C}$ lorsque $E$ est mis en dualité avec l'espace vectoriel $\tilde{F}$ des éléments de $E^{*}$ dont la restriction à $X$ est continue, $X$ étant muni de la trace de $\sigma(E, F)$.

A priori, les notions de mesure conique sur $E$ portée par $X$ lorsque $E$ est muni de $\sigma(E, F)$, et lorsque $E$ est muni de $\sigma(E, \tilde{F})$, sont différentes; nous allons voir qu'en fait il n'en est rien grâce aux propositions 4 et 5 .

Nous noterons $(E, F)$ ou $(E, \tilde{F})$ pour indiquer que $E$ est muni de $\sigma(E, F)$ ou de $\sigma(E, \tilde{F})$; remarquons que tout élément de $M(E, \tilde{F})$ induit par restriction un élément de $M(E, F)$.

4. Proposition. Tout élément de $M(E, \tilde{F})$ porté par $X$ est bien déterminé par sa restriction à $h(E, F)$.

5. Proposition. Tout élément $\mu \in M(E, F)$ porté par $X$ se prolonge univoquement en un élément $\mu \in M(E, \tilde{F})$ porté par $X$. L'application $\mu \rightarrow \tilde{\mu}$ est bijective linéaire, et positive.

Ces deux propositions résultent du lemme suivant.

6. LEMME. Soit $\mu \in M^{+}(E, F)$ portée par $X$; pour toute $f \in h(E, \tilde{F}), \forall \varepsilon>0$, il existe $u, v \in h(E, f)$ tels que $u \leqslant f \leqslant v$ sur $X$ et $\mu(v-u)<\varepsilon$.

Preuve. Il suffit de prouver le lemme avec $f$ convexe. Si $f=\sup \left(f_{i}\right), f_{i} \in \tilde{F}_{i}$, $1 \leqslant i \leqslant n$, prenons $\forall i, u_{i}, v_{i} \in F$ tels que $\forall i, u_{i} \leqslant f_{i} \leqslant v_{i}$ sur $X$, et $v_{i}(r(\mu))-$ $u_{i}(r(\mu))<\varepsilon / n$ de tels $u_{i}, v_{i}$ existent, à cause du théorème de séparation de Hahn-Banach, appliqué dans l'espace $E \times R$ au convexe fermé $((x, k) ; x \in X$, $\left.k \geqslant f_{i}(x)\right)$ et au point

$$
\left(r(\mu), f_{i}(r(\mu))-\varepsilon / 2 n\right),
$$


puis au convexe fermé $\left((x, k) ; x \in X, k<f_{i}(x)\right)$ et au point

$$
\begin{gathered}
\left(r(\mu), f_{i}(r(\mu))+\varepsilon / 2 n\right) ; \\
u=\sup \left(u_{i}\right) \quad \text { et } \quad v=\sup \left(v_{i}\right), \quad 1<i<n,
\end{gathered}
$$

répondent à la question puisque l'on $a, \operatorname{sur} X$,

$$
v-u<\sum_{1}^{n}\left(v_{i}-u_{i}\right)
$$

\section{REMARQues.}

$1^{\circ}$ Il résulte alors des deux propositions précédentes que les e.v.r. constitués par les éléments de $M(E, F)$ (resp. $M(E, \tilde{F})$ ) portés par $X$ sont canoniquement isomorphes, ce qui justifie la notation $M(X)$. Pour exprimer ce fait nous dirons que, si $X \in \mathcal{C}, M(X)$ ne dépend que du cône $X$ et de sa topologie.

$2^{\circ}$ Notons également que la notion d'ordre de Choquet sur $\mathrm{M}^{+}(X)$, associée à $S(E, F)$, est la même que celle associée à $S(E, \tilde{F})$ : étant donnés $f \in S(E, \tilde{F})$ et $\lambda, \mu \in M^{+}(X)$ avec $\lambda(g)<\mu(g), \forall g \in S(E, F)$, il existe grâce à 6 une suite $\left(f_{n}\right)$ de $S(E, F)$ avec $\lambda\left(f_{n}\right) \rightarrow \lambda(f)$ et $\mu\left(f_{n}\right) \rightarrow \mu(f)$. La notion d'ordre de Choquet, et donc aussi de mesure maximale, sur $M^{+}(X)$ ne dépend donc que du cône $X \in \mathcal{C}$ et de sa topologie; on notera $\max (X)$ l'ensemble des éléments maximaux de $M^{+}(X)$ pour cet ordre.

\section{L'intégrale supérieure des fonctions sous-linéaires et s.c.i.}

8. EXEMPLES.

$1^{\circ}$ Soit $E=L^{\infty}$ (classes de fonctions mesurables bornées sur $[0,1]$ muni de $d x$ ), muni de la topologie faible telle que $E^{\prime}=L^{1}, X=E^{+}$, et $\mu$ la mesure maximale sur $X$ qui représente la constante $1 \in X . \mu$ n'est pas de Daniell sur $\left.h(E)\right|_{X}$ :

$\forall k$ et $n$ entiers avec $1<k<2^{n}$, soit $e(k, n)$ le segment

$$
\left[(k-1) / 2^{n}, k / 2^{n}\right] \text { de }[0,1]
$$

soit $h_{n} \in h(E)$, définie par

$$
\forall f \in L^{\infty}, \quad h_{n}(f)=\sup _{1<k<2^{n}}\left(\int_{e(k, n)} f d x\right) ;
$$

on a $\left.h_{n}\right|_{X} \rightarrow 0$ en décroissant, et $\mu\left(h_{n}\right)=1, \forall n$.

$2^{\circ}$ Le disque unité $D$, considéré comme partie de $R^{2}$ et le cercle unité $U$ muni de la mesure de Haar, permettent de construire une mesure conique $\lambda$ sur $R^{3}$, portée par un élément $X \in \mathcal{S}$, telle que $\lambda$ ne soit pas de Daniell sur $\left.h\left(R^{3}\right)\right|_{K_{\lambda}}$ :

Soit $X \subset R^{3}$ de cône de $\delta$ admettant $D \times 1$ pour base; on a $R^{+} \cdot K_{\lambda} \backslash 0=\dot{X}$ (intérieur de $X$ ); noter qu'il existe une suite $\left(f_{n}\right)$ de fonctions continues définies 
sur $D$ telle que $f_{n}=1$ sur $U$, et $\left(f_{n}\right)$ décroit vers 0 sur

$$
\stackrel{\circ}{D}=D \backslash U \text {. }
$$

Soit $\mu \in M^{+}(X)$ avec $X \in \mathcal{C}$; nous allons chercher à prolonger $\mu$ à des fonctions définies sur $X$ et positivement homogènes, mais non nécessairement continues. Pour cela, le lemme suivant, du genre Fatou, est fondamental:

9. Lemme. Soient $E$ un espace faible, $X \in \mathcal{C}$ avec $X \subset E, \mu \in M^{+}(X)$, et $f \in h(E)$. Pour tout filtre $\mathscr{F}$ sur $S(E)$ tel que en tout point de $K_{\mu}$,

$$
f \leqslant \lim \inf (\mathscr{F}),
$$

on $a$

$$
\mu(f) \leqslant \lim \inf (\mu(\mathscr{F})) .
$$

Preuve. Soit $X_{1}, 1 \leqslant i \leqslant n$, une famille de cônes polyédraux ([4], 20.1 définition) recouvrant $X$, telle que, $\forall i, \exists \tilde{f}_{i} \in E^{\prime}$ avec $\left.f\right|_{X_{i}}=\left.\left(\tilde{f}_{i}\right)\right|_{X_{i}}$; une telle famille existe ([4], 30.2 proposition).

$\forall i, \exists \mu_{i} \in M^{+}\left(X_{i}\right), 1 \leqslant i \leqslant n$, avec $\mu=\sum \mu_{i} ;$ soit $x_{i}=r\left(\mu_{i}\right) ;$ on a

$$
x_{i} \in X_{i} \cap K_{\mu} .
$$

$\forall \varepsilon>0, \exists F_{e} \in \mathscr{F}$ tel que $g\left(x_{i}\right) \geqslant f\left(x_{i}\right)-\varepsilon / n$ pour tout $i$, et $\forall g \in F_{e}$. D'après le théorème de Hahn-Banach, $\forall h \in S(E)$, on a:

$$
\mu(h)=\sum \mu_{i}(h) \geq \sum h\left(x_{i}\right)
$$

et $\forall g \in F_{e}$

$$
\mu(g) \geqslant \sum f\left(x_{i}\right)-\varepsilon=\mu(f)-\varepsilon
$$

d'où le lemme.

10. Notation. Soient $E$ un espace faible, $X \in \mathcal{C}$ avec $X \subset E$; on note $C_{l}(X)$ la famille des fonctions sous-linéaires et s.c.i., définies sur $X$ et à valeurs dans $R \cup(+\infty)$.

Cette famille est stable par sup quelconque et par multiplication $\operatorname{par} \lambda>0$. Remarquons que $C_{i}(X)$ ne dépend que du cône $X$ et de sa topologie.

Nous aurons également besoin de la remarque suivante.

11. Proposition. Soit $E$ un espace faible, $X \in \mathcal{C}$ avec $X \subset E$; toute

$$
f \in C_{i}(X)
$$

est limite filtrante croissante sur $X d^{\prime}$ 'éléments de $S(E)$.

Preuve. Remarquons que, dans l'espace produit $E \times R$, le sur-graphe $G$ de $f$ $(G=((x, \lambda) ; x \in X$ et $\lambda \geqslant f(x)))$ est un cône convexe fermé. Si $x \in X$ est tel 
que $f(x)<+\infty$, il suffit de séparer strictement $G$ et $(x, f(x)-\varepsilon)$ pour tout $\varepsilon>0$, par un hyperplan fermé, à l'aide du théorème de Hahn-Banach.

Si $f(x)=+\infty$, on séparera strictement $G$ et le segment ayant pour extrémités $(0,-1)$ et $(x, k)$ pour tout $k \in R$.

12. Définition. Soient $E$ un espace faible, $X \in \mathcal{C}$ avec $X \subset E$, et $\mu \in$ $M^{+}(X)$; pour toute $f \in C_{i}(X)$, on pose

$$
\mu^{*}(f)=\sup (\mu(g) ; g \in S(E) \text { et } g \leqslant f \text { sur } X) .
$$

On a $\mu^{*}(\lambda f)=\lambda \mu^{*}(f), \forall \lambda>0$. Notons que $\mu^{*}(f) \in R \subset(+\infty)$.

Grâce à la proposition suivante, qui résulte du Lemme 9 , l'application $\mu^{*}$ ne dépend que du cône $X$ et de sa topologie.

13. Proposition. Soient $E$ un espace faible, $X \in \mathcal{C}$ avec $X \subset E$, et

$$
\mu \in M^{+}(X) \text {. }
$$

Pour toute $f \in C_{i}(X)$ et tout filtre $\mathscr{F}$ sur $S(E)$, admettant une partie formée d'éléments $\leqslant f$ sur $K_{\mu}$, et tel que $\mathcal{F} \rightarrow f$ sur $K_{\mu}$, on a

$$
\mu^{*}(f)=\lim (\mu(\mathscr{F})) \text {. }
$$

14. Corollaire. $\forall f, g \in C_{i}(X)$, on $a$

$$
\left(f \leqslant g \text { sur } K_{\mu}\right) \Rightarrow\left(\mu^{*}(f) \leqslant \mu^{*}(g)\right) .
$$

$\forall f, g \in C_{i}(X)$, on $a$

$$
\mu^{*}(f+g)=\mu^{*}(f)+\mu^{*}(g) .
$$

$\forall \mu, \nu \in M^{+}(X)$, on $a$

$$
(\mu+\nu)\left(f^{*}\right)=\mu\left(f^{*}\right)+\nu\left(f^{*}\right), \quad \forall f \in C_{i}(X) .
$$

Pour toute famille $f_{i}$ d'éléments $\geqslant 0$ de $C_{i}(X)$, on a $\mu^{*}\left(\sum f_{i}\right)=\Sigma \mu^{*}\left(f_{i}\right)$.

15. Notations. Soient $E$ un espace faible, et $\mu \in M^{+}(E)$; on note $C_{i}(\mu)$ la famille des fonctions sous-linéaires et s.c.i., définies sur $R^{+} \cdot K_{\mu}$, à valeurs dans $R \cup(+\infty)$, minorées par un élément (non fixé) de $E^{\prime}$. Comme $\forall X \in \mathcal{C}$, tel que $X \subset E$ et $\mu \in M^{+}(X)$, tout élément de $C_{i}(\mu)$ est la restriction à $R^{+} \cdot K_{\mu}$ d'un élément de $C_{i}(X)$, on peut donc parler, $\forall f \in C_{i}(\mu)$, par abus de langage, de $\mu^{*}(f)$.

Soit $C_{i}^{l}(\mu)=\left(f ; f \in C_{i}(\mu)\right.$ et $\left.\mu^{*}(f)<\infty\right)$.

16. Proposition. Soit $f \in C_{i}^{1}(\mu) ; \forall x \in K_{\mu}$, on a $f(x)<\mu^{*}(f)$.

Preuve. C'est une conséquence immédiate de la Proposition 13. 
17. Proposition. Soient $E$ un espace faible, $X \in \mathcal{C}$ avec $X \subset E$ et

$$
\mu \in M^{+}(X)
$$

soient $f \in C_{i}(\mu)$ et $f_{1} \in C_{i}^{\prime}(\mu)$; pour tout filtre $\mathcal{F}$ sur $C_{i}(\mu)$ tel que, en tout point de $K_{\mu}, f-f_{1} \leqslant \lim \inf (\mathcal{F})$, on $a$

$$
\mu^{*}(f)-\mu^{*}\left(f_{1}\right)<\lim \inf \left(\mu^{*}(\mathscr{F})\right) \text {. }
$$

Preuve. En raisonnant sur $f$ et sur le filtre $\left(\mathscr{F}+f_{1}\right)=(F ; \exists \tilde{F} \in \mathscr{F}$ avec $F=\tilde{F}+f_{1}$ ), on voit, grâce au Corollaire 14 , qu'il suffit de démontrer le résultat de l'énoncé avec $f_{1}=0$; de plus, d'après la définition 12 , il suffit d'envisager le cas où $f \in h(E)$.

Cela étant, la démonstration s'effectue exactement comme au Lemme 9, en tenant compte du Corollaire 14 et de la Proposition 16:

Soit $\left(\mu_{i}\right), 1 \leqslant i \leqslant n$, une famille finie d'éléments de $M^{+}(E)$ telle que, en posant $x_{i}=r\left(\mu_{i}\right), 1 \leqslant i \leqslant n$, on ait

$$
\mu=\sum \mu_{i} \quad \text { et } \quad \mu_{i}(f)=f\left(x_{i}\right), \quad 1 \leqslant i \leqslant n\left(\text { cf. } n^{\circ} 9\right) \text {; }
$$

$\forall g \in C_{i}(\mu)$, on a (cf. $\left.n^{\circ} 14\right) \mu^{*}(g)=\Sigma \mu_{i}^{*}(g)$, d'où

$$
\left.\mu^{*}(g)>\sum g\left(x_{i}\right) \quad \text { (cf. } n^{\circ} 16\right) \text {. }
$$

$\forall \varepsilon>0$ donné, $\exists F_{\varepsilon} \in \mathscr{F}$ tel que $\forall g \in F_{e}, g\left(x_{i}\right)>f\left(x_{i}\right)-\varepsilon / n, 1<i<n$, d'où $\mu^{*}(g) \geqslant \mu(f)-\varepsilon$.

18. Définition. Soient $E$ un espace faible, et $\mu \in M^{+}(E)$; on pose

$$
\Lambda^{1}(\mu)=C_{i}^{1}(\mu)-C_{i}^{1}(\mu)
$$

c'est un e.v.r. de fonctions sur $R^{+} \cdot K_{\mu}$; l'application $\mu^{*}$ se prolonge canoniquement, grâce au Corollaire 14, en un élément $>0$ de $\Lambda^{1}(\mu)^{*}$, noté encore $\mu^{*}$. On munit $\Lambda^{1}(\mu)$ de la semi-norme $p(f)=\mu^{*}(|f|)$. Notons que $p(|f|)=p(f)$ et que, $\forall f, g \geqslant 0$ dans $\Lambda^{1}(\mu)$, on a $p(f+g)=p(f)+p(g)$.

19. Proposition. Soient $X \in \mathcal{C}$ et $\mu \in \max (X)$; alors $\Lambda^{\prime}(\mu)$ muni de $p(f)$ est un espace complet.

(La réciproque est fausse; prendre une somme finie de mesures coniques ponctuelles.)

PreUve. Comme $h(E)$ est partout dense dans $\Lambda^{\prime}(\mu)$, il suffit de prouver que, pour toute suite $\left(h_{n}\right)$ de $h(E)$ avec $p\left(h_{n}\right)<1 / 2^{n}, \forall n$, la série $\sum h_{n}$ converge: soit $h_{n}=h_{n}^{+}-h_{n}^{-}$; prouvons la convergence de $\Sigma h_{n}^{+}: \mu$ étant maximale, on a $p\left(h_{n}^{+}-\check{h}_{n}^{+}\right)=0\left(\right.$ Si $f \in h(E), \check{f}$ désigne le plus grand élément de $C_{i}(X)$ tel que $\check{f} \leqslant\left. f\right|_{X}$ ); or $\sum \check{h}_{n}^{+} \in C_{i}(X)$; de plus (cf. $n^{\circ} 14$ )

$$
\mu^{*}\left(\sum \check{h}_{n}^{+}\right)=\sum \mu^{*}\left(\check{h}_{n}^{+}\right)=\sum \mu^{*}\left(h_{n}^{+}\right)<\sum 1 / 2^{n},
$$


d'où $\sum \check{h}_{n}^{+} \in C_{i}^{1}(\mu) ; \forall k$ entier, on a

$$
p\left(\sum \check{h_{n}^{+}}-\sum_{1}^{k} \check{h}_{n}^{+}\right)=\mu^{*}\left(\sum_{k+1}^{\infty} \check{h}_{n}^{+}\right)<1 / 2^{k}
$$

il y a donc convergence de $\Sigma h_{n}^{+}$vers $\Sigma \check{h}_{n}^{+}$au sens de $p$.

20. Remarque. Munissons $\Lambda^{1}(\mu)$ de la semi-norme $N$, définie par $N(f)=$ $\inf \left(\mu^{*}\left(f_{1}\right)+\mu^{*}\left(f_{2}\right) ; f_{1}, f_{2} \in C_{i}(\mu), f_{1}, f_{2} \geqslant 0\right.$ et $\left.f=f_{1}-f_{2}\right)$; on démontrerait que $\Lambda^{1}(\mu)$, muni de $N$, est un Banach.

Soient $E$ un espace faiblement complet, $X \subset E$ avec $X \in \mathcal{C}$, et $\mu \in M^{+}(X)$; $\mu$ étant de Daniell sur $h(E)$ ([4] 38.13), on peut développer la théorie de l'intégrale de Daniell ([1] et [5] \$61), et chercher à comparer cette théorie avec ce qui précède.

Notons que la fonction $\varphi_{X}$, définie sur $E$, valant 0 sur $X$ et $+\infty$ ailleurs, est dans $C_{i}(E)$, et que $\mu^{*}\left(\left.\varphi_{X}\right|_{X}\right)=0$.

21. Proposition. Lorsque $\varphi_{X}$ est $\mu$-intégrable pour la théorie de Daniell, alors $\mu$ est de Daniell sur $\left.h(E)\right|_{X}$. De plus, lorsque $X \in \mathcal{S}, E$ est isomorphe à $R^{N}$.

Preuve. Lorsque $\varphi_{X}$ est $\mu$-intégrable pour la théorie de Daniell, alors l'ensemble $(E \backslash X)$, où $\varphi_{X}=\infty$, est $\mu$-négligeable au sens de ([1] 6, et [5] \$61); on a donc ([1] 7, et [5] \$61) pour toute suite $\left(f_{n}\right)$ de $h(E)$ qui $\rightarrow 0$ en décroissant, sauf peut-être sur $(E \backslash X), \mu\left(f_{n}\right) \rightarrow 0$, ce qui est le résultat cherché.

Toute fonction $\mu$-intégrable pour la théorie de Daniell est nulle sur un s.e.v. de $E$ de codimension dénombrable, et donc au moins sur une droite de $E$ lorsque $E$ n'est pas isomorphe à $R^{N}$; dans ce cas, $\varphi_{X}$ ne peut pas être $\mu$-intégrable si $X \in \mathcal{S}$, car alors $X$ contiendrait une droite sur laquelle $\varphi_{X}$ serait nulle.

Nous allons voir que $\Lambda^{\prime}(\mu)$ peut être regardé comme un sous-espace vectoriel réticulé de $L^{1}(\mu)$ (espace classique déduit de $h(E)$ et de $\mu$ à l'aide de la théorie de Daniell, où deux éléments $f$ et $g$ tels que $\mu(|f-g|)=0$ sont identifiés). Nous dirons que $f, g \in \Lambda^{1}(\mu)$ sont $\mu$-équivalents lorsque

$$
\mu^{*}(|f-g|)=0 \text {. }
$$

22. Proposition. $\forall f \in C_{i}^{1}(\mu)$ la famille des fonctions de $C_{i}^{1}(\mu), \mu$-équivalentes $\grave{a} f$, admet un plus grand élément pour l'ordre usuel des fonctions.

Preuve. D'après la Proposition 17, il suffit de prouver que $\left(u, v \in C_{i}^{1}(\mu)\right.$ avec $u, v \mu$-équivalentes à $f) \Rightarrow(\sup (u, v) \mu$-équivalente à $f)$. Or on a

$$
u+v=\sup (u, v)+\inf (u, v)>\sup (u, v)+w,
$$


où $w$ est l'élément de $C_{i}^{1}(\mu)$ ainsi obtenu: on se place dans $E \times R$; soit $\Gamma_{\mu}=\left(R^{+} \cdot K_{\mu}\right) \times R$ et $\Gamma_{f}$ le sur-graphe de $f$

$$
\Gamma_{f}=\left((x, \lambda) ; x \in R^{+} \cdot K_{\mu} \text { et } \lambda>f(x)\right) ;
$$

$\Gamma_{u}, \Gamma_{v}$ et $\Gamma_{f}$ sont trois sous-cônes convexes, fermés, relatifs de $\Gamma_{\mu}$, et on a $\Gamma_{u}+\Gamma_{v} \subset \Gamma_{f} ; w$ sera caractérisée par

$$
\Gamma_{w}=\left(\overline{\Gamma_{u}+\Gamma_{v}}\right) \cap \Gamma_{\mu}
$$

comme $f \in C_{i}^{1}(\mu)$, on a $f \leqslant w$ puisque $\Gamma_{w} \subset \Gamma_{f}$; et on a $f \in C_{i}^{1}(\mu)$; comme aussi $w \leqslant u$ et $w \leqslant v$, on a $\mu^{*}(w)=\mu^{*}(f)$.

On a donc $\mu^{*}(u)+\mu^{*}(v) \geqslant \mu^{*}(\sup (u, v))+\mu^{*}(w)$; d'où

$$
2 \mu^{*}(f)>\mu^{*}(\sup (u, v))+\mu^{*}(f) \text {, }
$$

soit $\mu^{*}(\sup (u, v))=\mu^{*}(f)$ puisque $\sup (u, v) \geqslant f$.

La proposition suivante explicite la comparaison de $\Lambda^{1}(\mu)$ et de $L^{1}(\mu)$.

23. Proposition. Il existe une injection canonique de $\Lambda^{1}(\mu)$, modulo la $\mu$ équivalence, dans $L^{\prime}(\mu)$. Plus précisément:

$1^{\circ}$ Soit $f=C-C^{\prime}$ avec $C, C^{\prime} \in C_{i}^{1}(\mu)$; soient $\left(C_{n}\right)$ et $\left(C_{n}^{\prime}\right)$ deux suites croissantes de $S(E)$ telles que $C_{n} \leqslant C, C_{n}^{\prime} \leqslant C^{\prime}$ sur $K_{\mu}$, et

$$
\mu\left(C_{n}\right) \rightarrow \mu^{*}(C), \quad \mu\left(C_{n}^{\prime}\right) \rightarrow \mu^{*}\left(C^{\prime}\right) .
$$

A la classe de $f$ dans $\Lambda^{1}(\mu)$ correspond la classe de $\lim \left(C_{n}\right)-\lim \left(C_{n}^{\prime}\right)$ dans $L^{1}(\mu)$.

$2^{\circ}$ Réciproquement, soient $\left(C_{n}\right)$ et $\left(C_{n}^{\prime}\right)$ deux suites croissantes de $S(E)$ telles que $\lim \left(C_{n}\right)<\infty$ et $\lim \left(C_{n}^{\prime}\right)<\infty$. Soit $g$ une fonction sur $E$ telle que $g=\lim \left(C_{n}\right)-$ $\lim \left(C_{n}^{\prime}\right)$ en tout point où ces limites sont $<\infty$. La classe de $g$ (qui est dans $L^{1}(\mu)$ ) est l'image de la classe dans $\Lambda^{\prime}(\mu)$ de la restriction de $g$ à $R^{+} \cdot K$.

Preuve. On utilisera l'abréviation $L$ pour "limite", dans les formules.

Pour le $1^{\circ}$, il faut vérifier que $\forall S, S^{\prime} \in C_{i}^{\prime}(\mu)$ et $\forall\left(S_{n}\right),\left(S_{n}^{\prime}\right)$, suites croissantes de $S(E)$ telles que $S_{n} \leqslant S, S_{n}^{\prime} \leqslant S^{\prime}$ sur $K_{\mu}$, et

$$
\mu\left(S_{n}\right) \rightarrow \mu^{*}(S), \quad \mu\left(S_{n}^{\prime}\right) \rightarrow \mu^{*}\left(S^{\prime}\right),
$$

on a

( $f$ et $S-S^{\prime}$ sont $\mu$-équivalentes dans $\Lambda^{l}(\mu)$ )

$$
\Rightarrow\left(L\left(C_{n}\right)-L\left(C_{n}^{\prime}\right) \text { et } L\left(S_{n}\right)-L\left(S_{n}^{\prime}\right) \text { sont égales dans } L^{1}(\mu)\right) \text {. }
$$

Par abus de langage, on notera encore $\mu$ le prolongement canonique de $\mu$ à 
$L^{1}(\mu)$; on a alors

$$
\begin{aligned}
\mu\left(\mid\left(L\left(C_{n}\right)-\right.\right. & \left.\left.L\left(C_{n}^{\prime}\right)\right)-\left(L\left(S_{n}\right)-L\left(S_{n}^{\prime}\right)\right) \mid\right) \\
= & 2 \mu\left(\sup \left(L\left(C_{n}\right)+L\left(S_{n}^{\prime}\right), L\left(C_{n}^{\prime}\right)+L\left(S_{n}\right)\right)\right) \\
& -\mu\left(L\left(C_{n}\right)+L\left(S_{n}^{\prime}\right)+L\left(C_{n}^{\prime}\right)+L\left(S_{n}\right)\right) \\
= & \lim \left(2 \mu\left(\sup \left(C_{n}+S_{n}^{\prime}, C_{n}^{\prime}+S_{n}\right)\right)-\mu\left(C_{n}+S_{n}^{\prime}+C_{n}^{\prime}+S_{n}\right)\right) \\
= & 2 \mu^{*}\left(\left.\sup \left(L\left(C_{n}\right)+L\left(S_{n}^{\prime}\right), L\left(C_{n}^{\prime}\right)+L\left(S_{n}\right)\right)\right|_{R^{+} \cdot K_{\mu}}\right) \\
& -\mu^{*}\left(\left.\left(L\left(C_{n}\right)+L\left(S_{n}^{\prime}\right)+L\left(C_{n}^{\prime}\right)+L\left(S_{n}\right)\right)\right|_{R^{+} \cdot K_{p}}\right) .
\end{aligned}
$$

Pour prouver que la première partie de l'expression précédente vaut

$$
2 \mu^{*}\left(\sup \left(C+S^{\prime}, C^{\prime}+S\right)\right) \text {, }
$$

il suffit d'utiliser la Proposition 13 et la remarque suivante:

$\forall A, B, a, b \in C_{i}{ }^{1}(\mu)$ avec $a \leqslant A, b \leqslant B$ et $\mu^{*}(a)=\mu^{*}(A), \mu^{*}(b)=\mu^{*}(B)$, on a,

$$
\mu^{*}(\sup (A, B))=\mu^{*}(\sup (a, b)) \text {, }
$$

car $\sup (A, B)-\sup (a, b) \leqslant(A-a)+(B-b)$.

Pour achever de prouver ce $1^{\circ}$, il suffit de remarquer que l'on a

$$
\begin{aligned}
0= & \mu^{*}\left(\left|\left(C-C^{\prime}\right)-\left(S-S^{\prime}\right)\right|\right)=2 \mu^{*}\left(\sup \left(C+S^{\prime}, C^{\prime}+S\right)\right) \\
& -\mu^{*}\left(C+S^{\prime}+C^{\prime}+S\right) .
\end{aligned}
$$

Le $2^{\circ}$ est évident, une fois prouvé $1^{\circ}$.

\section{L'intégrale supérieure des fonctions sous-linéaires}

24. Notations. Soient $E$ un espace faiblement complet, $\mu \in M^{+}(E), X \subset E$ tel que $X \in \mathcal{C}$, et $\mu \in M^{+}(X)$. Dans tout ce qui suit, $Y$ désignera un cône convexe de $E$ tel que:

$1^{\circ} K_{\mu} \subset Y \subset X$.

$2^{\circ} Y$ est une partie héréditaire de $X$ pour le préordre induit par $X . C_{Y}$ désignera la famille des fonctions sous-linéaires, définies sur $Y$, à valeurs dans $R \cup(+\infty)$, minorées par un élément (non fixé) de $E^{\prime}$.

25. Définition. $\forall f \in C_{Y}$, on pose

$$
\mu_{Y}^{*}(f)=\inf \left(\mu^{*}(g) ; g \in C_{i}(X) \text { et } f \leqslant g \text { sur } Y\right) \text {. }
$$

Cette définition a toujours un sens, car la fonction valant $+\infty$ sur $X \backslash 0$, et 0 en 0 , est dans $C_{i}(X)$ et majore tous les éléments de $C_{Y}$; on a

$$
\mu_{Y}^{*}(f) \in R \cup(+\infty),
$$

car $f$ est minoré par un élément de $E^{\prime}$ sur $Y$.

$\mu_{Y}^{*}$ ne dépend que du cône $X$ et de sa topologie, $\mu$ et $Y$ étant fixés, mais $\mu_{Y}^{*}$ ne dépend pas du cône $X, E, E^{\prime}, \mu$ et $Y$ êtant fixés, vérifiant les conditions de 24 . 
26. EXEMPLES (éclairant la définition 25).

$1^{\circ}$ (Notations de $8,2^{\circ}$ ) Soit $f$ une fonction affine, défine sur $R^{+} \cdot K_{\lambda}$, telle que $f>1$ sur $\dot{D} \times 1$; on a $\lambda_{X}^{*}(f)>0$. Or, $\forall \varepsilon>0$, il existe une suite $\left(f_{n}\right) \mathrm{de}-S\left(R^{3}\right)$, $>0$ et croissant vers $f$ sur $K_{\lambda}$, telle que $\lambda\left(f_{n}\right)<\varepsilon, \forall n$.

Il suffit de construire les restrictions des $f_{n}$ à $D \times 1$ : soit $\varphi$ une fonction affine $>0$ sur $D ; \forall \varepsilon>0$, construisons une suite croissante $\left(\varphi_{n}\right)$ de fonctions de $-S\left(R^{2}\right)$ telle que $0 \leqslant \varphi_{n} \leqslant \varepsilon$ sur $U$ et $\varphi_{n} \rightarrow \varphi$ sur $D^{\circ}=D \backslash U$. Soit $\left(g_{n}\right)$ une suite auxiliaire de fonctions concave continues sur $D$ telle que $\left(g_{n}\right)$ soit croissante, $g_{n}<\varphi$ partout sur $D, g_{n}=0$ sur $U$, et $g_{n} \rightarrow \varphi$ sur $\dot{D}$; on prendra $\varphi_{1} \in-S\left(R^{2}\right)$ telle que $g_{1} \leqslant \varphi_{1}<\varphi$ sur $D$, et $\varphi_{1}<\varepsilon / 2$ sur $U$; soit $\hat{g}_{2}$ la plus petite fonction concave et s.c.s. définie sur $D$, telle que $\hat{g}_{2}>g_{2}$, et $\varphi_{1}$; on a $\hat{g}_{2}<\varphi$ sur $D$, et $\hat{g}_{2}=\varphi_{1}$ sur $U$, car dans $D \times R^{+}$, le sous-graphe de $\hat{g}_{2},((x, t) ; x \in D$ et $0<t \leqslant \hat{g}_{2}(x)$ ), est l'enveloppe convexe des sous-graphes de $g_{2}$ et $\varphi_{1}$; comme $\left.\hat{g}_{2}\right|_{U}=\left.\varphi_{1}\right|_{U}$ est continue, $\exists \varphi_{2} \in-S\left(R^{2}\right)$ telle que $\hat{g}_{2}<\varphi_{2}<\varphi$ sur $D$, et $\varphi_{2}<$ $\varepsilon / 2+\varepsilon / 4$ sur $U$.

Supposons construits les $\varphi_{1}, \ldots, \varphi_{n} \in-S\left(R^{2}\right)$ tels que $\varphi_{i}<\varphi_{j}$, pour $1<i<$ $j<n$ et, pour $1 \leqslant i<n, \varphi>\varphi_{i} \geqslant g_{i}$ sur $D$, et $0<\varphi_{i}<\varepsilon / 2+\cdots+\varepsilon / 2^{i}$ sur $U$ : construisons $\varphi_{n+1}:$ soit $\hat{g}_{n+1}$ la plus petite fonction concave et s.c.s. sur $D$ telle que $\hat{g}_{n+1} \geqslant g_{n+1}$ et $\varphi_{n}$; on a, comme pour $\hat{g}_{2}, \hat{g}_{n+1}<\varphi$ sur $D$, et $\hat{g}_{n+1}=\varphi_{n}$ sur $U$; donc $\exists \varphi_{n+1} \in-S\left(R^{2}\right)$ telle que $\hat{g}_{n+1}<\varphi_{n+1}<\varphi$ sur $D$, et $\varphi_{n+1}<\varepsilon / 2$ $+\cdots+\varepsilon / 2^{n+1}$ sur $U$. Il est clair que $\left(\varphi_{n}\right)$, ainsi construite, va être une suite de $-S\left(R^{2}\right)$ telle que $0 \leqslant \varphi_{n} \leqslant \varepsilon$ sur $U$, et $\left(\varphi_{n}\right)$ croît vers $\varphi$ sur $\dot{D}$.

$2^{\circ}$ Il existe un convexe compact mêtrisable $X$, que l'on considèrera comme la base d'un cône localement compact, une fonction $f$ convexe et borélienne définie sur $X$, une mesure de Radon $\mu>0$ dont le support contient $\mathcal{E}(X)$ (ensemble des points extrémaux de $X)$, de sorte que $\mu(f)<\inf (\mu(g) ; g>f, g$ convexe s.c.i. sur $X$ ): on prendra pour $X$ l'ensemble des mesures de Radon $>0$ et de masse 1 sur $[0,1]$, pour $\mu$ la mesure maximale sur $X$ représentant l'élément $(d x) \in X$, et pour $f$ l'application définie par $\forall m \in X, f(m)=m_{d}(1)$ ( $m_{d}$ : partie diffuse de $m$ ), on a $\mu(f)=0$, et $f(r(\mu))=1$; on voit que $f$ est borélienne en notant que, $\forall k \in R$,

$$
\begin{aligned}
(m ; m \in X & \text { et } 1-f(m)>k) \\
& =\bigcup_{p \in N, q \in N}\left(m ; \exists x_{1}, \ldots, x_{p} \in[0,1] \text { et } \exists \alpha_{1}, \ldots, \alpha_{p}>0\right. \\
& \text { tels que } \left.m>\sum_{1}^{p} \alpha_{i} \delta_{x_{i}} \text { et } \sum_{1}^{q} \alpha_{i}>k+1 / q\right) \\
= & \bigcup_{p \in N, q \in N} K_{p, q},
\end{aligned}
$$

car chaque $K_{p, q}$ est une partie compacte de $X$.

$f$ convient grâce à la Proposition 16 . 
27. Proposition. $\forall f, g \in C_{Y},(f \leqslant g) \Rightarrow \mu_{Y}^{*}(f) \leqslant \mu_{Y}^{*}(g)$.

$\forall f, g \in C_{Y}$, on $a \mu_{Y}^{*}(f+g)<\mu_{Y}^{*}(f)+\mu_{Y}^{*}(g)$.

$\forall f \in C_{Y}$ et $\forall \lambda>0$, on $a \mu^{*}(\lambda f)=\lambda \mu_{Y}^{*}(f)$.

28. Proposition. Soient $\lambda, \mu \in M^{+}(X)$ avec $X \in \mathcal{E}$, et $\left(K_{\lambda} \cup K_{\mu}\right) \subset Y$; on a $(\lambda+\mu)_{Y}^{*}=\lambda_{Y}^{*}+\mu_{Y}^{*}$.

Preuve. Il est évident que $(\lambda+\mu)_{Y}^{*} \geqslant \lambda_{Y}^{*}+\mu_{Y}^{*}$ à cause du Corollaire 14. Soit $f \in C_{Y}$; il suffit d'envisager le cas où $\lambda_{Y}^{*}(f)+\mu_{Y}^{*}(f)<\infty$;

$$
\forall \varepsilon>0, \exists u, v \in C_{i}(X)
$$

avec $f<u$ et $v$ sur $Y$, et avec $\lambda^{*}(u)<\lambda_{Y}^{*}(f)+\varepsilon, \mu^{*}(v)<\mu_{Y}^{*}(f)+\varepsilon$. Il suffit alors de trouver $w \in C_{i}(X)$ avec $f \leqslant w \leqslant u$ et $v$ sur $Y$; déterminons $w$ à l'aide de son sur-graphe $\Gamma_{w}$ dans $X \times R$ : rappelons que le sur-graphe $\Gamma_{u}$ de $u$ dans $X \times R$ est défini par $((x, k) ; x \in X$ et $f(x) \leqslant k)$; on a $\Gamma_{u}$ et $\Gamma_{v} \in \mathcal{S} ; \Gamma_{u}$ et $\Gamma_{v}$ sont contenus dans un même cône de $\delta$ ([3] chap. II, $\$ 6 n^{\circ} 8$, lemme 1 et prop. 11); $w$ sera caractérisée par $\Gamma_{w}=\Gamma_{u}+\Gamma_{v}$; on a donc $\Gamma_{w} \in \delta$ ([3] chap. II, $\$ 6 n^{\circ}$ 8, prop. 11) et $w \in C_{i}(X)$; de plus, comme $Y$ est une partie héréditaire de $X$ pour le préordre défini par $X$, la fonction $w$ est $\geqslant f$ sur $Y$. $w$ est bien l'élément cherché.

Comme nous ne considérerons plus qu'une seule mesure conique $\mu$, on peut supposer que $X=\overline{R^{+} \cdot K_{\mu}}$, car alors $X \in \mathcal{C}$, et $X$ porte $\mu$ ([4] 38.5).

29. Lemme. Lorsque $X \in \mathcal{\delta}$, pour toute suite croissante $\left(f_{n}\right)$ d'éléments de $C_{Y}$, on $a$

$$
\mu_{Y}^{*}\left(\lim \left(f_{n}\right)\right)=\lim \left(\mu_{Y}^{*}\left(f_{n}\right)\right) .
$$

Preuve. Il est clair que $\mu_{Y}^{*}\left(\lim \left(f_{n}\right)\right)>\lim \left(\mu_{Y}^{*}\left(f_{n}\right)\right)$; de plus si $\lim \left(\mu_{Y}^{*}(f)\right)=$ $+\infty$, le résultat est évident. Supposons donc $\lim \left(\mu_{Y}^{*}\left(f_{n}\right)\right)<+\infty$. Soit $\varepsilon>0$; $\forall n>1, \exists h_{n} \in C_{i}(X)$ telle que $f_{n}<h_{n}$ sur $Y$, et $\mu^{*}\left(h_{n}\right)<\mu_{Y}^{*}\left(f_{n}\right)+\varepsilon / 2^{n}$; posons $g_{n}=\sup \left(h_{1}, h_{2}, \ldots, h_{n}\right)$; on a $g_{n} \in C_{i}(X), f_{n}<g_{n}$ sur $Y$, et $\left(g_{n}\right)$ est une suite croissante; montrons que $\forall n \geqslant 1$,

$$
\mu^{*}\left(g_{n}\right) \leqslant \mu_{Y}^{*}\left(f_{n}\right)+\varepsilon\left(1-1 / 2^{n}\right) .
$$

Une fois prouvée cette inégalité, on en déduit que, $\forall n>1$,

$$
\mu^{*}\left(g_{n}\right) \leqslant \lim \left(\mu_{Y}^{*}\left(f_{n}\right)\right)+\varepsilon,
$$

et en posant $g_{0}=\lim \left(g_{n}\right)$, on a, comme $\left(g_{n}\right)$ est une suite croissante et grâce à la Proposition 17,

$$
\mu^{*}\left(g_{0}\right) \leqslant \lim \left(\mu_{Y}^{*}\left(f_{n}\right)\right)+\varepsilon ;
$$

d'où le lemme, car on a, sur $Y, g_{0}>\lim \left(f_{n}\right)$ et $g_{0} \in C_{i}(X)$. 
Pour prouver que, $\forall n \geqslant 1, \mu^{*}\left(g_{n}\right) \leqslant \mu_{Y}^{*}\left(f_{n}\right)+\varepsilon\left(1-1 / 2^{n}\right)$, on procède par récurrence; le cas $n=1$ étant évident, supposons l'inégalité établie pour l'entier $n$; on a

$$
g_{n+1}=\sup \left(g_{n}, h_{n+1}\right)
$$

soit $\varphi$ l'élément de $C_{i}(X)$ dont le sur-graphe $\Gamma_{\varphi}$ est l'enveloppe convexe des sur-graphes $\Gamma_{g}$ et $\Gamma_{h}$ de $g_{n}$ et de $h_{n+1}$ ( $\varphi$ existe grâce à des arguments déjà invoqués en 28: $\Gamma_{g}$ et $\Gamma_{h}$ sont contenus dans un même cône de $\delta$, et $\Gamma_{\varphi}=\Gamma_{g}+$ $\Gamma_{h} \in \mathcal{S}$ ). Comme $Y$ est une partie héréditaire de $X$, pour le préordre défini par $X$, on a $\varphi \geqslant f_{n}$ sur $Y$, et $g_{n+1}+\varphi \leqslant g_{n}+h_{n+1}$; d'où:

$$
\mu^{*}(\varphi)+\mu^{*}\left(g_{n+1}\right) \leqslant \mu^{*}\left(g_{n}\right)+\mu^{*}\left(h_{n+1}\right)
$$

et

$$
\mu_{Y}^{*}\left(f_{n}\right)+\mu^{*}\left(g_{n+1}\right) \leqslant \mu_{Y}^{*}\left(f_{n}\right)+\varepsilon\left(1-\left(1 / 2^{n}\right)\right)+\mu_{Y}^{*}\left(f_{n+1}\right)+\varepsilon / 2^{n+1} ;
$$

ce qui est l'inégalité visée.

30. Remarque. Dans 28,29 , on peut éviter de supposer que $X \in \mathcal{\delta}$, mais il feut alors supposer que les adhérences des graphes des fonctions considérées ne contiennent pas de droite: cela permet en effet d'utiliser, dans les preuves, des sur-graphes de fonctions qui sont des cônes de $\delta$.

\section{Bibliographie}

[1] R. Becker, 'Mesures coniques et intégrale de Daniell', C. R. Acad. Sci. Paris, t. 283, 1976, p. 611-614.

[2] N. Bourbaki, Topologie générale, Chap. 1 et 4, Nouvelle édition (Hermann, Paris, 1971).

[3] __ Espaces vectoriels topologiques, Chap. 1 et 2, 2e édition (Hermann, Paris, 1963) (Acta. scient. et ind., 1189 a; Bourbaki, 15).

[4] G. Choquet, Lectures on analysis, vol. 1-3. (New York, Amsterdam, W. A. Benjamin, 1969 Mathematics Lecture Note Series).

[5] F. Riesz et B. Sz. Nagy, Lecons d'analyse fonctionelle, 5e édition. (Paris, Gauthier-Villars, 1968).

Equipe d'Analyse, Tour 46

Université Pierre et Marie Curie

4 place Jussieu

75230 Paris Cedex 05

France 